\title{
Quality Assessment of Oenological Tannins Utilising Global Selectivity Chemical Sensors Array ("Electronic Tongue")
}

\author{
J.-L. Puech ${ }^{1}$, A. Prida ${ }^{2 *}$ and S. Isz ${ }^{3}$
}

(1) Unité Mixte de Recherche "Science pour l'OEnologie”, Institut National de la Recherche Agronomique, 2 place Viala, 34060 Montpellier, France.

(2) Tonnellerie Seguin Moreau, Z.I. Merpins - B.P. 94, 16103, Cognac, France.

(3) Alpha M.O.S. France, 20 Didier Daurat Avenue 31400 Toulouse, France.

Submitted for publication: October 2006

Accepted for publication: July 2007

Key words: Oenological tannins, electronic tongue, flavour attributes, bitterness, astringency.

\begin{abstract}
Oenological tannin is a common name for food additives containing tannins utilised in winemaking practices. The main taste feature of oenological tannin is the taste sensation of astringency and bitterness. In the present paper, samples of various oenological tannins (oak, chestnut, gall, tara, querbacho, grape seed and grape skin tannins) were analysed by means of a tasting panel, measuring the flavour attributes bitterness, astringency, body, duration of flavour and similarity with wine tannins, and using the array of global selectivity chemical sensors (electronic tongue) " $\alpha$-ASTREE" Liquid and Taste Analyzer (Alpha M.O.S., Toulouse, France). Principal component analysis of the electronic tongue outputs applied for different tannin solutions provides good discrimination according to their chemical nature. Consequently, three main classes of oenological tannins, namely gallotannins, ellagitannins and condensed tannins, could be identified and separated. The global output of the electronic tongue is quite responsive to changes in the bitterness and astringency of model quinine and alum solutions and, once calibrated (correlation coefficients of $0.976(p<0.001)$ and 0.996 ( $p<0.001)$ respectively) could quantify their concentrations with good precision. The electronic tongue output was found to be correlated with the flavour attributes of oenological tannins. The best correlation was observed for bitterness. This fact could be explained by more constant calibration and lesser influence of any interfering factors on this attribute.
\end{abstract}

\section{INTRODUCTION}

Polyphenolic molecules (tannins) are essential components for the sensorial qualities of wines, such as taste, astringency and colour. They originate mainly from grapes during maceration and fermentation.

Oenological tannin is a common name for food additives containing tannins that are produced by the extraction of different botanical raw materials and utilised in winemaking practices. These preparations contain polyphenolic fractions belonging to different chemical classes of tannins, namely condensed tannins, prepared from Vitis vinifera grapes (seeds and skins) and querbacho; ellagic tannins, prepared from oak and chestnut materials; and gallic tannins, from exotic wood preparations (Vivas, 1997).

The principal use of oenological tannins is to eliminate potentially unstable protein fractions. However, oenological tannins also can modify some of the organoleptic properties of wines: red colour stabilisation in red wines, wine astringency, bitterness and blending, and improve the structure of wine (Marquette, 1999; Poinsaut, 2000; Lurton et al., 2002). Therefore, chemical analyses, as well as sensory assessments, are required for the quality control of oenological tannins.

The main taste features of oenological tannins are the taste sensation of bitterness and the tactile sensation of astringency. Oenological tannin solutions and wines rich in tannins are often described using complex attributes like body and persistence or duration of flavour. Although the body of wine is generally correlated with sug- ar, alcohol, glycerol and, obviously, with the tannin content of the wines, the intensity of the wine fragrance also appears to enhance the perception of body. Duration of taste is the length of time the wine or tannin solutions maintain their distinctive characteristics, thus it is correlated with the specificity and persistence of tannin taste. Human panels can analyse these features using different techniques (Robichaud \& Noble, 1990; Lee \& Lawless, 1991; Vivas, 1997; Brossaud et al., 2001). However, tasting panels are limited by various subjective factors, such as taste saturation and fatigue. These parameters can cause inconsistent judgments and incorrect applications. Furthermore, the tasting panel method requires a considerable number of trained and highly qualified panellists, along with a very careful tasting procedure.

The so-called "electronic tongue" is an analytical method comprising the analysis of liquid samples using an array of chemical sensors and mathematical signal treatments. This system closely mimics the working principle of the human taste sense. The simplicity, rapidity and possibility of clear interpretation of data are the quality characteristic of this method. Taste threshold concentrations of the liquid sensors exceed human taste sensations by a two-fold factor (Tan et al., 2001a, b). Electronic tongue applications are not restricted only by water solutions. It has been shown that the electronic tongue operates rather well in low- and highalcohol solutions such as beer (Tan et al., 2001a, b), wine (Legin et al., 1999; Di Natale et al., 2000) and spirits (Iiyama et al., 1996; Legin et al., 2005). 
Previously, Iiyama et al. (1995) and Puech et al. (2003) reported that the electronic tongue signal is correlated with the astringent intensity of the purified chemicals catechin, chlorogenic and gallic acid, as well as with natural mixtures such as tannic acid and oak heartwood extract.

In the present paper, electronic tongue analysis was used to assess various oenological tannins and taste standards of the pure chemicals alum (aluminium potassium sulphate) and quinine chloride. The instrumental quantification was compared to the evaluation of the tasting panel.

\section{MATERIALS AND METHODS}

\section{Sample preparation}

Tannins were obtained by extraction from different botanical raw materials, viz. oak (Quercus sp.), chestnut (Castanea sativa), gallnut (Quercus infectoria), tara (Cesalpina spinosa), quebracho (Quebracho sp.), grape seed and grape skin (Vitis vinifera), were investigated. The specific characteristics of these tannins, and the particularities of their use, have been described by Vivas (1997). The samples were received directly from producers and distributors of oenological products (Table 1). The pure chemicals, alum and quinine, were obtained from Fluka. Aqueous solutions of oenological tannins with a concentration of $0.5 \mathrm{~g} / 1$ were used for both the instrumental analysis and the taste assessment. This concentration was chosen because most panellists prefer to evaluate taste attributes at their middle intensity. All solutions were prepared from dry chemicals just before analysis.

\section{Descriptive analysis}

Five attributes were chosen to characterise the taste of tannin solutions: bitterness, astringency, body, duration of flavour and similarity to wine tannins. The intensity of the latter reveals how close the perception of oenological tannin is to the perception of natural tannin in red wine. The intensities of the taste attributes for the oenological tannin solutions were determined by a tasting panel composed of highly skilled oenologists (seven persons: three males and four females) and graded according to the 10-point scale, where 0 denotes low intensity and 10 denotes a high intensity. The panel was selected because of its homogeneity and on the basis of the correct responses of the individuals during preliminary tasting (Peleg et al., 1998). The tasting panel was trained by using standard aqueous solutions (alum and quinine) representing different bitterness and astringency intensities, as well as different red wines in order to reach agreement regarding other taste attributes. The oenological tannins were evaluated in two replicates (two panel sessions;

\section{TABLE 1}

Sources of tannins.

\begin{tabular}{ll}
\hline Materials & Suppliers \\
\hline Oak tannin & Oenotan \\
Chestnut tannin & OenoFrance \\
Gall tannin & Laffort \\
Tara tannin & Martin Vialatte \\
Quebracho tannin & Littorale Enologie \\
Grape seed tannin & Ferco Enologie \\
Grape skin tannin & Ferco Enologie \\
Alum & Fluka \\
Quinine hydrochloride & Fluka \\
\hline
\end{tabular}

one replicate per session). Simple rinsing with water between each two samples was performed. The middle intensity of astringency/ bitterness and the reasonable number of samples (seven samples per session) ensured that sensory fatigue was avoided. The results obtained for the intensity of each attribute were processed as follows: 1 . centring stage - using the mean value of intensity for each tasting session; and 2. reducing stage - using the standard deviation of intensity for each taste session. The values obtained and centred at 0 were then re-centred at 1 by adding 1 to the values in order to simplify the further analysis. The one-way ANOVA was carried out for processed results from the panellists.

\section{“a-ASTREE Electronic Tongue" analysis}

The “ $\alpha$-ASTREE” Liquid and Taste Analyser (Electronic Tongue) (Alpha M.O.S., Toulouse, France) consists of an array of 7 liquid cross-sensitive electrodes, a 16-position autosampler and an associated interface electronic module. The electrodes are sensors - chemical field effect transistors. Each sensor (ZZ, BA, BB, CA, GA, HA, JB) is composed of a silicon transistor (an ion-sensitive field effect transistor generally used for $\mathrm{pH}$ detection) with an organic coating that governs the sensitivity and selectivity of each individual sensor (Bergveld, 1970; Frolov et al., 1997). This set, referred to as $\alpha$-ASTREE Sensor Array \#1, was designed to provide suitable characteristics and to permit the differentiation of the majority of food (Tan et al., 2001a,b). A measurement consists of the potentiometric difference for each sensor and the $\mathrm{Ag} / \mathrm{AgCl}$ reference electrode in the equilibrium state at room temperature. Therefore, an integral signal for each sample comprised a vector with seven individual sensor determinations. Four measurements were performed for each sample; the average and standard deviation were calculated.

\section{Statistical analysis of instrumental results}

The PCA (principal component analysis) method was used to treat the output from the sensor array as a means to visualise the tannin groups. Each sensor's output constitutes a different component of the variables.

The partial least square (PLS) statistical method was applied to correlate the global output of the electronic tongue (linear combination of the output of each sensor) with the concentration of standard substances (alum, quinine), as well as with the sensorial notes of tasting panel.

Statistical treatments were carried out using SPSS 10 for Windows (SPSS Inc.) and Alpha M.O.S. built-in software (Alpha M.O.S., France).

\section{RESULTS AND DISCUSSION}

\section{Tasting panel}

An ANOVA was performed for taste intensities (centred and reduced notes) using the Student-Newman-Keuls test. The average taste intensities, standard deviations reported by the panel and Fisher's coefficients are shown in Table 2. A high variation was reported between the evaluations of the different tasters. Nevertheless, the sensorial data allow several tannins to be separated from the others: 1. tara tannin, characterised by low intensity of all attributes except bitterness; 2 - quebracho and gall nut tannin, characterised by high bitterness, astringency and duration of flavour. Surprisingly, the oak and chestnut tannins were evaluated as being the most similar to wine tannins. In contrast, grape seed and grape skin tannins were evaluated as being less similar to 
wine tannins. This possibly is linked to the high purification of grape-derived tannins, which lose their olfactory and taste hints characteristic of wine tannins, although oak and chestnut tannins are perceived as being well matched to barrel-aged wines.

It is worth mentioning that the majority of these attributes are cross-correlated (Table 3). The strongest correlation is between body and astringency, which emphasises the similarity in sensorial appreciation of these attributes by the panellists. It is possible that in these binary systems (water and tannin), contrary to the case with real wine, the sensation of body arises only from the total quantity of phenolic compounds, which is also the origin of astringency.

\section{PCA}

The PCA treatment of the signals from the electronic tongue (data matrix is 7 direct sensor outputs $\times 7$ tannins $\times 4$ replications) groups the samples according to their chemical nature, namely as condensed tannins, gallotannins and ellagitannins (Fig. 1). The axis of the first principal component approximately expresses the whole total variability $(98.61 \%)$. This axis permits the grouping of tannins into classes due to their chemical structures.

\section{Correlation of electronic tongue instrument with taste standards}

In this series of experiments, the electronic tongue was applied to different solutions of taste-reference substances, namely alum for astringency and quinine hydrochloride for a bitter taste. The PLS model was designed to correlate the overall output of the electronic tongue with the concentration of the reference substances (data matrix is 7 direct sensor outputs $x 4$ concentrations $x 4$ replications).

The curves are plotted in Figs 2 and 3, and express excellent correlations between the aforementioned parameters. The Pearson correlation coefficients were $0.976(\mathrm{p}<0.001)$ for quinine and $0.996(\mathrm{p}<0.001)$ for alum. Averages, standard deviations assessed by the electronic tongue and the difference between real and assessed concentrations were calculated. The low standard deviations (0.0006-0.001 g/L for quinine solution; 0.009-0.043 g/L for alum solution) show the stability of output from the sensor. The residual mean squares for the linear regressions of quinine concentration versus quinine concentration and alum concentration versus alum concentration, both assessed instrumentally, are $10^{-6}$ and 0.0006 , respectively. This stresses the reliability of the current calibrations.

As has been shown, the determinations by the electronic tongue displayed excellent correlation with the quinine/alum input data. The models could be used successfully for quinine/alum determination in unknown samples.

\section{Correlation between instrumental and sensorial data}

The data from the electronic tongue were correlated with sensory data for each taste attribute using the PLS model (data matrix is 7 direct sensor outputs $\times 7$ tannins $x 3$ replications). The descriptive statistical data of this determination are shown in Table 4.

TABLE 2

Sensorial taste intensities of the tannin solutions (centred and reduced notes).

\begin{tabular}{|c|c|c|c|c|c|c|c|c|c|c|}
\hline \multirow[t]{2}{*}{ Tannin } & \multicolumn{2}{|c|}{ Bitterness } & \multicolumn{2}{|c|}{ Astringency } & \multicolumn{2}{|c|}{ Body } & \multicolumn{2}{|c|}{$\begin{array}{l}\text { Duration of } \\
\text { flavour }\end{array}$} & \multicolumn{2}{|c|}{$\begin{array}{l}\text { Similarity to } \\
\text { wine tannins }\end{array}$} \\
\hline & Mean & St. dev. & Mean & St. dev. & Mean & St. dev. & Mean & St. dev. & Mean & St. dev. \\
\hline Chestnut & $1.04^{\mathrm{a}}$ & 0.98 & $1.25^{\mathrm{a}, \mathrm{b}}$ & 1.07 & $0.89^{\mathrm{a}, \mathrm{b}}$ & 0.57 & $1.03^{\mathrm{a}, \mathrm{b}}$ & 0.69 & $1.40^{\mathrm{b}}$ & 0.77 \\
\hline Oak & $1.05^{\mathrm{a}}$ & 1.09 & $0.85^{\mathrm{b}}$ & 1.12 & $1.33^{\mathrm{b}}$ & 1.00 & $0.96^{\mathrm{a}, \mathrm{b}}$ & 1.11 & $1.23^{\mathrm{a}, \mathrm{b}}$ & 1.27 \\
\hline Gall & $0.72^{\mathrm{a}}$ & 0.54 & $1.34^{\mathrm{b}}$ & 1.14 & $1.39^{\mathrm{b}}$ & 1.09 & $1.15^{\mathrm{b}}$ & 0.84 & $1.07^{\mathrm{a}, \mathrm{b}}$ & 1.07 \\
\hline Grape skin & $0.46^{\mathrm{a}}$ & 0.55 & $0.75^{\mathrm{a}, \mathrm{b}}$ & 0.79 & $0.62^{\mathrm{a}, \mathrm{b}}$ & 0.53 & $0.58^{\mathrm{a}, \mathrm{b}}$ & 0.49 & $0.78^{\mathrm{a}, \mathrm{b}}$ & 0.99 \\
\hline Grape seed & $0.33^{\mathrm{a}}$ & 0.64 & $0.54^{\mathrm{a}, \mathrm{b}}$ & 0.86 & $0.47^{\mathrm{a}, \mathrm{b}}$ & 0.88 & $0.74^{\mathrm{a}, \mathrm{b}}$ & 0.92 & $0.57^{\mathrm{a}, \mathrm{b}}$ & 0.93 \\
\hline Quebracho & $2.88^{b}$ & 0.85 & $1.44^{\mathrm{b}}$ & 0.78 & $0.97^{\mathrm{a}, \mathrm{b}}$ & 0.96 & $2.04^{\mathrm{c}}$ & 0.96 & $0.60^{\mathrm{a}, \mathrm{b}}$ & 0.70 \\
\hline Tara & $0.57^{\mathrm{a}}$ & 0.79 & $0.14^{\mathrm{a}}$ & 0.76 & $0.25^{\mathrm{a}}$ & 0.83 & $0.17^{\mathrm{a}}$ & 0.84 & $0.27^{\mathrm{a}}$ & 0.71 \\
\hline F (probab.) & \multicolumn{2}{|c|}{$17,136(* * *)$} & \multicolumn{2}{|c|}{$3,533(* *)$} & \multicolumn{2}{|c|}{$3,483(* *)$} & \multicolumn{2}{|c|}{$6,552(* * *)$} & \multicolumn{2}{|c|}{$2,574(*)$} \\
\hline
\end{tabular}

Note: Means with same letters in a column are not significantly different (ANOVA).

$*-\mathrm{p}<0,05$

$* *-\mathrm{p}<0,01$

$* * *-p<0,001$

TABLE 3

Correlation between the sensory attributes assessed in this study.

\begin{tabular}{|c|c|c|c|c|c|}
\hline & Bitterness & Astringency & Body & $\begin{array}{l}\text { Duration of } \\
\text { flavour }\end{array}$ & $\begin{array}{l}\text { Similarity with } \\
\text { wine tannins }\end{array}$ \\
\hline Bitterness & 1 & $0.44 * *$ & $0.25^{*}$ & $0.58 * *$ & 0.04 \\
\hline Astringency & & 1 & $0.68 * *$ & $0.56 * *$ & $0.30 * *$ \\
\hline Body & & & 1 & $0.62 * *$ & $0.61 * *$ \\
\hline Duration of flavour & & & & 1 & $0.31 * *$ \\
\hline Similarity with wine tannins & & & & & 1 \\
\hline
\end{tabular}

Note:

** - Correlation is significant at the 0.01 level (2-tailed).

* - Correlation is significant at the 0.05 level (2-tailed). 


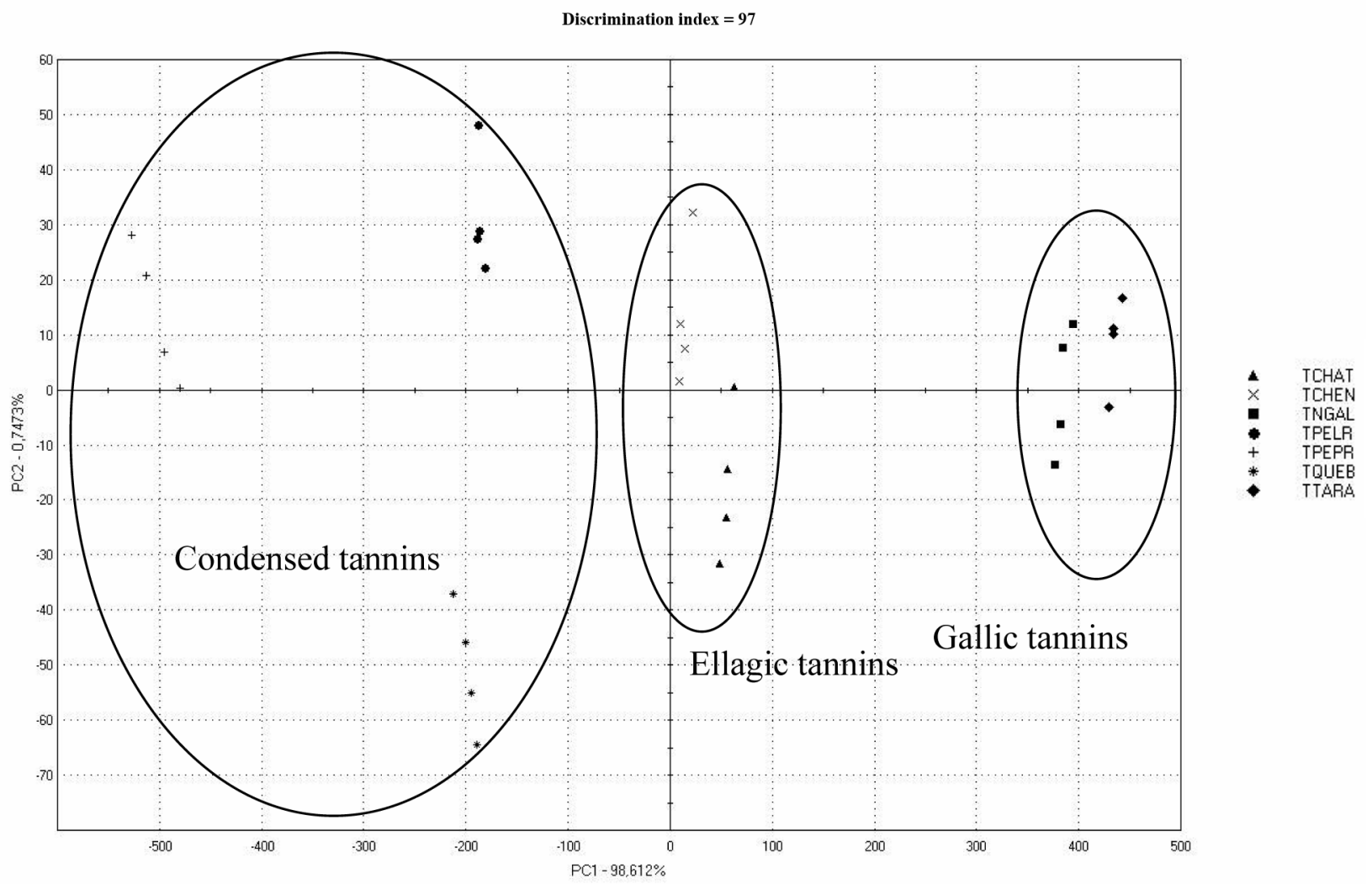

FIGURE 1

Principal Component Analysis (PCA) of the electronic tongue variables. Individual plots.

Note : TCHAT - chestnut tannin, TCHEN - oak tannin, TNGAL - gal tannin, TPELR - grape skin tannin, TPERP - grape seed tannin, TQUEB - quebracho tannin, TTARA - tara tannin

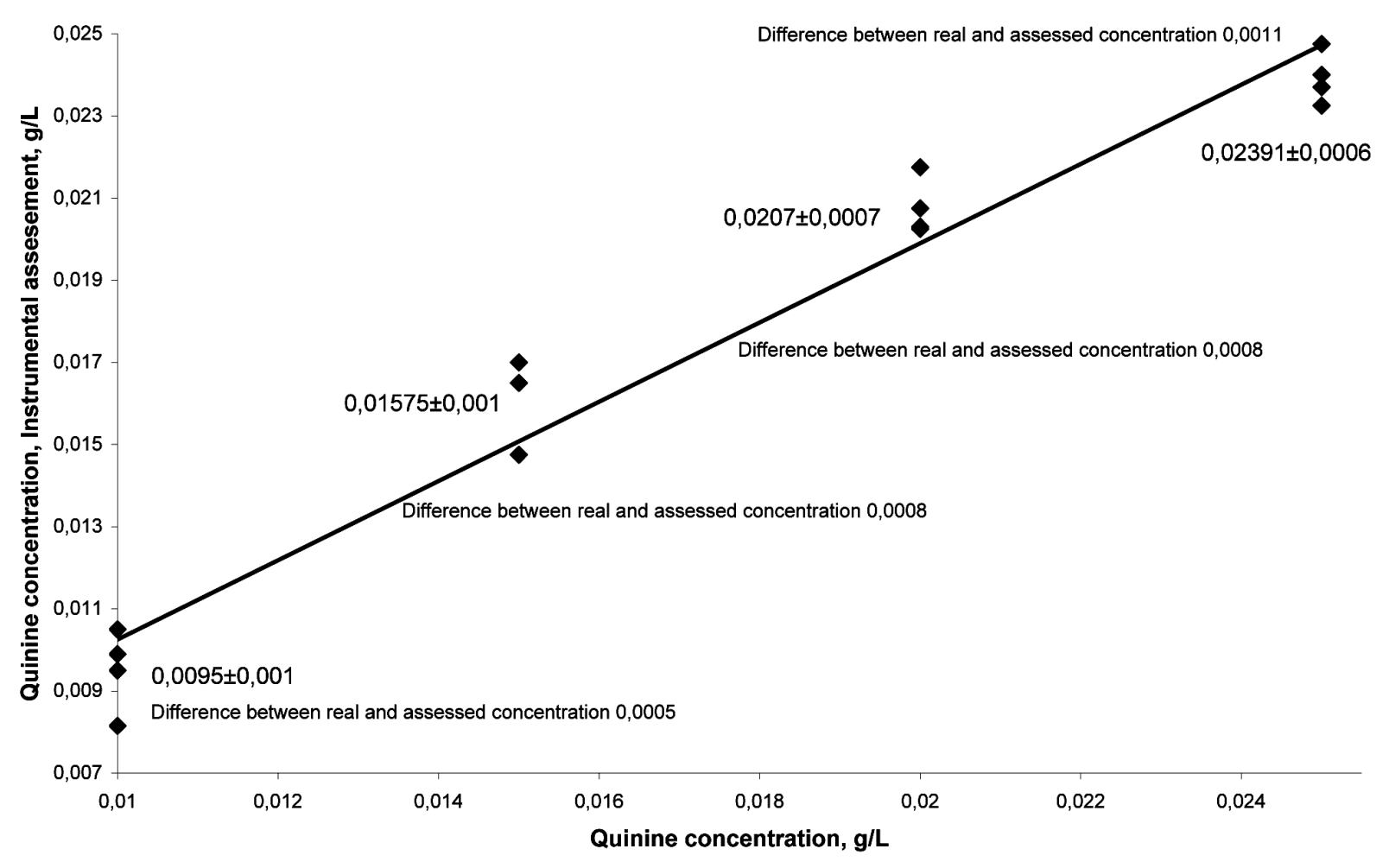

FIGURE 2

Calibration of the intensity of bitterness with different concentrations of quinine. 


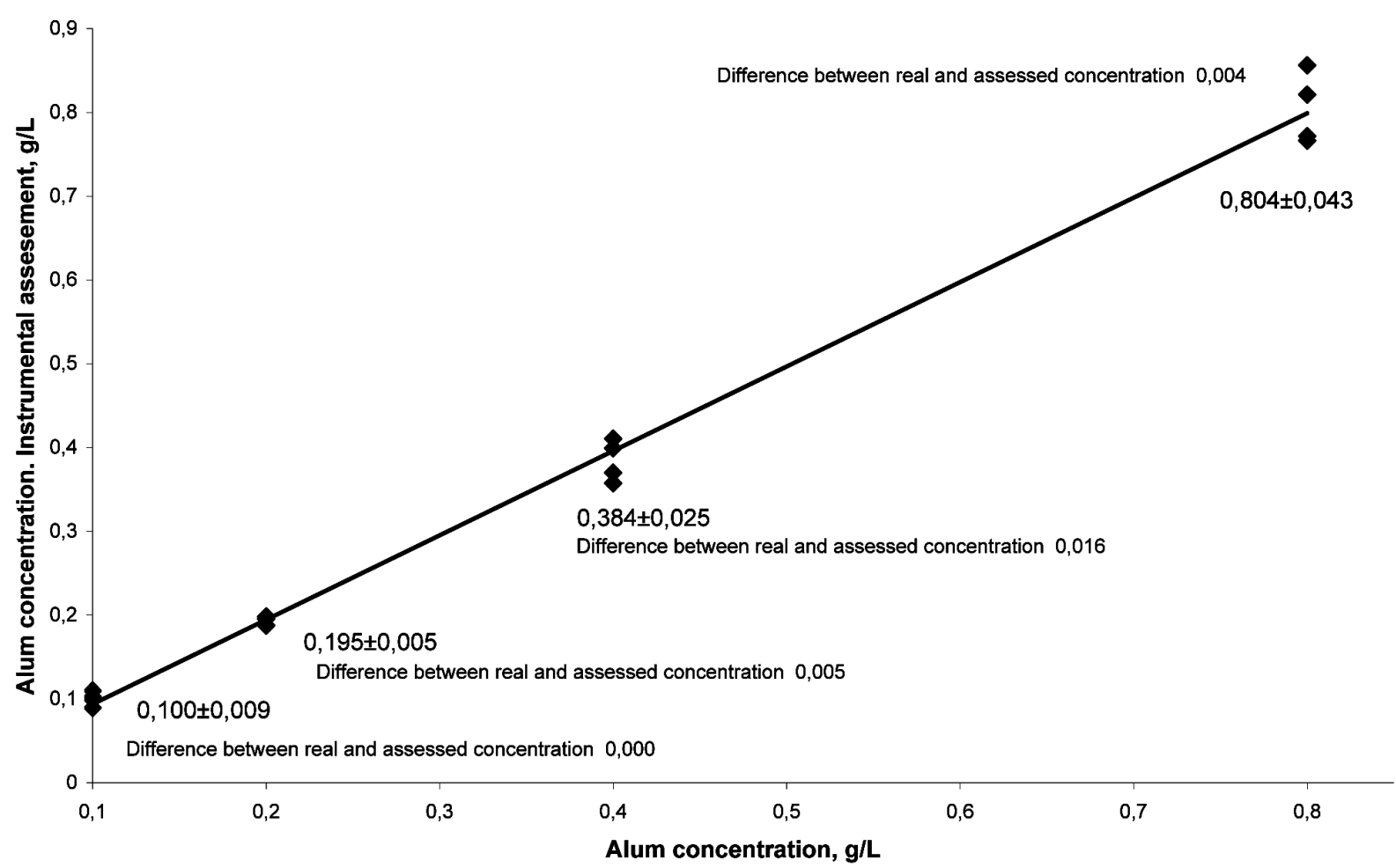

FIGURE 3

Calibration of the intensity of astringency with different concentrations of alum.

\section{TABLE 4}

Instrumental taste intensities of the tannin solutions.

\begin{tabular}{|c|c|c|c|c|c|c|c|c|c|c|c|c|c|c|c|}
\hline \multirow[t]{2}{*}{ Tannin } & \multicolumn{3}{|c|}{ Bitterness } & \multicolumn{3}{|c|}{ Astringency } & \multicolumn{3}{|c|}{ Body } & \multicolumn{3}{|c|}{$\begin{array}{l}\text { Duration of } \\
\text { flavour }\end{array}$} & \multicolumn{3}{|c|}{$\begin{array}{l}\text { Similarity to } \\
\text { wine tannins }\end{array}$} \\
\hline & Mean & St.dev. & Dif. & Mean & St.dev. & Dif. & Mean & St.dev. & Dif. & Mean & St.dev. & Dif. & Mean & St.dev. & Dif. \\
\hline Chestnut & 1.28 & 0.14 & 0.24 & 1.39 & 0.10 & 0.13 & 1.28 & 0.09 & 0.05 & 1.39 & 0.15 & 0.36 & 1.27 & 0.05 & 0.13 \\
\hline Oak & 0.79 & 0.13 & 0.27 & 0.98 & 0.10 & 0.14 & 1.03 & 0.08 & 0.14 & 0.89 & 0.15 & 0.07 & 1.21 & 0.04 & 0.01 \\
\hline Gall & 0.72 & 0.09 & 0.01 & 0.84 & 0.08 & 0.50 & 0.90 & 0.08 & 0.49 & 0.73 & 0.12 & 0.42 & 0.83 & 0.03 & 0.24 \\
\hline Grape skin & 0.55 & 0.08 & 0.09 & 0.74 & 0.05 & 0.01 & 0.76 & 0.07 & 0.14 & 0.66 & 0.09 & 0.07 & 1.06 & 0.06 & 0.28 \\
\hline Grape seed & 0.34 & 0.02 & 0.01 & 0.48 & 0.10 & 0.06 & 0.34 & 0.11 & 0.13 & 0.76 & 0.12 & 0.02 & 0.43 & 0.06 & 0.15 \\
\hline Quebracho & 2.62 & 0.26 & 0.26 & 1.35 & 0.10 & 0.09 & 1.00 & 0.09 & 0.03 & 1.81 & 0.13 & 0.23 & 0.65 & 0.06 & 0.05 \\
\hline Tara & 0.59 & 0.05 & 0.01 & 0.52 & 0.07 & 0.39 & 0.61 & 0.08 & 0.36 & 0.45 & 0.09 & 0.28 & 0.47 & 0.06 & 0.20 \\
\hline Correlation coefficients & & 0.93 & & & 0.79 & & & 0.75 & & & 0.85 & & & 0.87 & \\
\hline
\end{tabular}

Note: Dif. - Difference with sensory data; St.dev. - standard deviation.

Excellent correlation was observed for bitterness (correlation coefficient 0.93 ) and quite good ones for other attributes. It is noteworthy that the standard deviation of the instrumental data and the difference between the sensory and instrumental data (Table 4) are considerably lower than the standard deviation of the sensory data (Table 2). The good correlation with the sensory data, as well as the low error of detection, allows the determination of attribute intensities of unknown tannins.

In this study we tested the robustness of analysis by designing a PLS model based on the instrumental data from three detections for each tannin being studied and a projection of the fourth one on the basis of this model in order to predict the intensities of their attributes. The data obtained are given in the Table 5. Excellent results were recorded for bitterness in the case of all the tan- nins, except for oak. In contrast, all other flavour attributes were recognised less well, with the average difference being roughly twice that for bitterness. There are two possible reasons for this fact. The first is related to the subjectivity of the tasting panel and the divergent evaluation of complex taste attributes by the different panellists. In fact, complex oenological terms such as body or similarity of wine tannins are not easy to describe. Therefore, simpler attributes, such as bitterness, which are more familiar to the panellists, receive more constant judgments and consequently correlate better with the electronic tongue. The second explanation could be linked to the fact that the sensors of the electronic tongue possibly are more sensitive to changes in the intensity of bitterness than to changes in other flavour attributes. It is well known that bitterness is perceived on the basis of the presence of 
TABLE 5

Difference between sensorial note and instrumental note on the basis of three detections.

\begin{tabular}{|c|c|c|c|c|c|}
\hline Tannin & Bitterness & Astringency & Body & Flavour length & $\begin{array}{l}\text { Similarity with } \\
\text { wine tannins }\end{array}$ \\
\hline Chestnut & 0.1005 & 0.2469 & 0.1495 & 0.6461 & 0.1814 \\
\hline Oak & 0.4809 & 0.0516 & 0.2882 & 0.1456 & 0.1352 \\
\hline Gall & 0.2237 & 0.3384 & 0.3276 & 0.1186 & 0.0073 \\
\hline Grape skin & 0.1143 & 0.266 & 0.2867 & 0.218 & 0.389 \\
\hline Grape seed & 0.0072 & 0.1748 & 0.0758 & 0.3385 & 0.119 \\
\hline Quebracho & 0.0812 & 0.2995 & 0.2407 & 0.1024 & 0.4736 \\
\hline Tara & 0.0693 & 0.5678 & 0.5211 & 0.5661 & 0.4129 \\
\hline Mean & 0.15 & 0.28 & 0.27 & 0.31 & 0.25 \\
\hline Maximum & 0.48 & 0.57 & 0.52 & 0.65 & 0.47 \\
\hline
\end{tabular}

a limited range of flavour-active phenolics, although other components interfere slightly. In contrast, astringency, body etc. are produced by a very wide range of chemicals, and their perception is influenced by other compounds occurring in the medium, as well as by the specific properties of the medium, such as density, viscosity and so on (Peleg et al., 1998; Smith et al., 1998; Courregelongue et al., 1999; Peleg and Noble, 1999; Smith and Nobel, 1999). As has been shown previously in the case of quinine, the output of the electronic tongue is correlated excellently with the concentration of this single taste-active compound. Thus, bitterness, as a less complex sensation, could easily be described using the electronic tongue, and this fact has been proved experimentally. The decreasing reliability of the determination of bitterness and astringency in a tannin solution in comparison to solutions of pure chemicals could be explained by the aforementioned influence of interfering factors relating to chemicals and the medium.

\section{CONCLUSIONS}

Using the electronic tongue technique permits the characterisation of oenological tannins from the point of view of chemical structure and taste. PCA application provides good discrimination between the three main classes of oenological tannins, namely gallotannins, ellagitannins and condensed tannins. The global output of the electronic tongue is quite responsive to changes in the bitterness and astringency of model quinine and alum solutions and, when calibrated, could quantify their concentration with good precision. The output of the electronic tongue was found to be correlated with the flavour attributes of oenological tannins. The best correlation was observed for bitterness. This fact could be explained by the more constant calibration and less of an influence from interfering factors on this attribute.

The use of the electronic tongue without any determination of individual substances can give a global response, and express the taste characteristics of tannin extracts. It is a rapid analytical method that does not need sample preparation. It could be considered as a prospective tool for the quality control and characterisation of tannins, and could replace complicated, expensive and subjective tasting panels and provide higher precision of determination.

\section{LITERATURE CITED}

Bergveld, P., 1970. Development of an ion-sensitive solid-state device for neurophysiological measurements, IEEE Trans. Biomed. Eng. BME 17, 70-71.

Brossaud, F., Cheynier, V. \& Noble, A., 2001. Bitterness and astringency of grape and wine polyphenols. Aust. J. Grape Wine Res. 7, 33-39.
Courregelongue, S., Schlich, P. \& Noble, A., 1999. Using repeated ingestion to determine the effect of sweetness, viscosity and oiliness on temporal perception of soymilk astringency. Food Qual. Pref. 10, 273-279.

Di Natale, C., Paolesse, R., Macagnano, A., Mantini, A., D’Amico, A., Ubigli, M., Legin, A., Lvova, L., Rudniskaya, A. \& Vlasov, Y., 2000. Application of a combined artificial olfaction and taste system to the quantification of relevant compounds in red wine Sensors \& Actuators B 69, 342-347.

Frolov, A., Choulga, A., Abalov, Y., Kononenko, L., Netchiporuk, V., Strikha, N., Jaffrezic, R. \& Martelet, C., 1997. Capteur de type ISFET dont le substrat est isolé électriquement. Brevet français 2(706), 616.

Iiyama, S., Suzuki, Y., Ezaki, S., Arikawa, Y. \& Toko, K., 1996. Objective scaling of taste of sake using taste sensor and glucose sensor. Materials Sci. Eng. C4, 45-49.

Lee, C. \& Lawless, H., 1991. Time-course of astringent sensations. Chem. Senses $16,3,225-238$

Legin, A., Rudnitskaya, A., Seleznev, B. \& Vlasov, Y., 2005. Electronic tongue for quality assessment of ethanol, vodka and eau-de-vie, Analytica Chimica Acta 534, 129-135.

Legin, A., Rudnitskaya, A., Vlasov, Y., Di Natale, C., Mazzone, E. \& D’Amico, A., 1999. Application of electronic tongue for quantitative analysis of mineral water and wine. Electroanalysis 11, 814-820.

Lurton, L., Laurent, M. \& Garnier, C., 2002. Vinification en rouge: des tanins de raisin pour stabiliser la couleur. Rev. Enol. 104, 27-28.

Marquette, B., 1999. Usage des tanins en œnologie. Rev. Fr. Oenol. 174, 26-28.

Peleg, H. \& Noble, A., 1999. Effect of viscosity, temperature, and pH on astringency in cranberry juice. Food Qual. Pref. 10, 343-347.

Peleg, H., Bodine, K. \& Noble A., 1998. The influence of acid on astringency of alum and phenolic compounds, Chem. Senses 23, 371-378.

Poinsaut, P., 2000. Les tanins oenologiques. Propriétés et applications pratiques. Rev. Enol. 97, 33-35.

Puech, J.-L., Prida, A. \& Isz, S., 2003. La langue électronique appliquée à la tonnellerie. Revue des Enologues 109S, 35-37.

Robichaud, J. \& Noble, A., 1990. Astringency and bitterness of selected phenolics in wine J. Sci. Food Agric. 53, 343-353.

Smith, A., June, H. \& Noble, A., 1998. Effects of viscosity on the bitterness and astringency of grape seed tannin. Food Qual. Pref. 7, 161-166.

Smith, A. \& Noble, A., 1999. Effects of increased viscosity on the sourness and astringency of aluminum sulfate and citric acid. Food Qual. Pref. 9(3), 139-144.

Tan, T., Schmitt, V. \& Isz, S., 2001a. Electronic tongue: a new dimension in sensory analysis. Food Technology 55(10), 44-60.

Tan, T., Schmitt, V., Isz, S. \& Saint Martin, E., 2001b. The electronic tongue: a time-saving tool for off-taste detection, bitterness masking study and gold reference assessment in the beverage industries. The European Food and Drink Review $3,65-75$.

Vivas, N., 1997. Composition et propriétés des préparations commerciales de tanins à usage oenologique. Rev. CEnol. 84, 15-21. 